\title{
Nonspecific Retroactive Interference in Children and Adults
}

\author{
Jillen Fatania ${ }^{1}$ and Tom Mercer $^{2}$ \\ ' University College London, UK \\ ${ }^{2}$ University of Wolverhampton, Wolverhampton, UK
}

ABSTRACT

Retroactive interference (RI) is a primary source of forgetting and occurs when new information disrupts or damages an existing memory. Prior research has shown that children are susceptible to RI when the to-be-remembered and interfering information are similar, but it is unclear whether they are also vulnerable to nonspecific RI. This form of interference occurs when a memory is disrupted by an unrelated and dissimilar distractor task, and the present study explored six- and seven-yearolds susceptibility to such nonspecific RI. In two experiments, participants learnt a list of words and completed a free recall test 5 min later. During the interval, participants either remained quiet (the control condition) or completed spot-the-difference puzzles (the interference condition). In Experiment 1, the children were highly susceptible to nonspecific interference, whereas a sample of adults were not affected by the interfering task. However, when a new sample of children were given more time to encode and retrieve the words in Experiment 2, they were able to resist interference. Nonspecific RI can damage children's memory, but they do have the ability to prevent this form of interference in certain circumstances.

retroactive interference, memory development, nonspecific interference, forgetting

\section{INTRODUCTION}

The ability to maintain information in memory is a crucial aspect of human cognition and an essential component of everyday tasks. Yet this also appears to be challenging, as the ability to retain memories typically declines over time (e.g., Murre \& Dros, 2015). Such timebased forgetting could be due to retroactive interference (RI), which occurs when new incoming information and stimuli interfere with an existing representation, leading to its loss.

RI is often cited as a source of forgetting (Nairne \& Pandeirada, 2008) and it may be most damaging when it takes place shortly after new learning (e.g., Dewar, Alber, Butler, Cowan, \& Della Sala, 2012; Dewar, Alber, Cowan, \& Della Sala, 2014; Mercer, 2015). This could be because new memories are especially vulnerable to RI before they have undergone consolidation (see Wixted, 2004, 2005, for a review). To be able to function effectively in daily life, however, some resistance to RI is essential. Indeed, RI may have a relatively mild effect on adults with an intact memory (e.g., Dewar, Cowan, \& Della Sala, 2010; Hardt, Nader, \& Nadel, 2013), but its effects are much more potent in other populations. This includes those suffering from anterograde amnesia brought about by injury, illness, or disease (see Dewar et al., 2010), and it may also include typically developing children.

Studies have investigated both children's susceptibly to different forms of RI (e.g., Levy-Gigi \& Vakil, 2010) and ways of reducing it (e.g., Howe, 2002, 2004). Other studies have tested whether children become more resistant to RI as they age-an idea which seems plausible given the developmental changes that occur to children's cognitive control processes (Davidson, Amso, Anderson, \& Diamond, 2006). Surprisingly, early evidence indicated that younger children were less susceptible to RI than older children (Koppenaal, Krull, \& Katz, 1964; Loomis \& Hall, 1968), but more recent experimental evidence has

Corresponding author: Tom Mercer, Millennium City (MC) Building, Faculty of Education, Health and Wellbeing, University of Wolverhampton, Wolverhampton, WV1 1LY. Phone: +44(0)1902 321368.

Email: t.mercer2@wlv.ac.uk 
found that RI effects are robust across age groups (Howe, 1995, 2002, 2004). Lee and Bussey (2001) provided a valuable demonstration of $\mathrm{RI}$ in children by using a version of the A-B A-C paradigm. Four- and seven-year-old children participated in a target game on one or three occasions. They were shown different sets of objects (clothing, fruit, and rooms in a toy house) and, during an initial recall test, were required to select the room in which a particular item of clothing belonged. Two days after the last target game, children in the experimental group completed an interpolated game either one or three times, whereas control participants did not. The interpolated game was identical to the target game, except a new set of fruit was used, hence, it was predicted to cause RI. The final phase of the study occurred three weeks later and involved another memory test. The children were asked to name the clothing that they could remember and then recall the fruit and the room associated with that item. Seven-year-old participants had more accurate levels of recall and fewer intrusion errors than four-year-old participants. Repeated participation in the target game also boosted memory performance, yet children of both ages were affected by the interpolated game. Specifically, this game decreased correct recall of objects from the target game and repeatedly participating in the target game did not protect memory from RI, highlighting its potent influence.

Other studies have compared RI effects in children and adults. Darby and Sloutsky (2015), for example, used an associate learning task to examine memory interference in children (aged four or five years old) and adults. In a computerised task, participants were presented with pairs of real world objects that moved into an occluder and disappeared from view. The objects then emerged either from the left or right side of the occluder, next to a cartoon character. The task was to predict where the object would reappear. Participants completed a training phase, followed by three experimental phases. In Experiment 1 , the first and third phases contained identical object pairings, whereas the second phase included unique pairings that served as a source of interference. Importantly, the phase two object pairings were either unique or had some degree of overlap with the objects shown in the first phase. For example, if participants associated the Objects A and B in Phase 1, the Phase 2 overlapping condition might include the pairing $\mathrm{A}$ and $\mathrm{C}$, whereas the unique condition would involve completely new associations (e.g., Objects I and J). To assess RI, performance at the end of Phase 1 was compared with that at the start of Phase 3. There was a significant interaction between phase and condition for children only, and planned comparisons indicated that children did worse in the overlapping condition, in comparison with the unique condition. This was taken to reflect evidence for RI in children, and when comparing accuracy using the first block of Phase 1 as a baseline, the children were quicker to relearn unique pairs at the start of Phase 3, but found it more difficult to relearn overlapping pairs. Darby and Sloutsky (2015) argued that this reflected catastrophic RI, whereas adults were more resilient to RI.

Findings like those of Darby and Sloutsky (2015) indicate that the ability to resist RI may improve as individuals age, yet the vast majority of studies examining RI in children have ensured that the to-be-re- membered stimuli and the interfering information were highly similar. Lee and Bussey (2001) designed their interpolated game to be almost identical to the target game, and RI in Darby and Sloutsky's (2015) experiment was determined by the overlap between learning in Phases 1 and 2. Such similarity-based interference has long been held to cause the most forgetting (McGeoch, 1931; Robinson, 1920), and this may apply to children, too (e.g., Darby \& Sloutsky, 2015; see also Levy-Gigi \& Vakil, 2010). Yet whilst similar RI may be particularly damaging, evidence from adult samples has shown that dissimilar interfering events can produce forgetting, too. This is known as nonspecific RI (Keppel, 1968; Wixted, 2004). Evidence for nonspecific RI was first reported by Müller and Pilzecker (1900) in one of the pioneering studies of forgetting, and Dewar, Cowan, and Della Sala (2007) replicated these early findings. They showed participants a list of 15 nouns for recall 8 min later. During the interval, participants in different groups were subjected to a specific type of interfering task. One group listened to a radio recording, another group viewed visual images for subsequent serial recall, whereas a third group detected differences between pairs of images. Participants in the fourth group completed a mathematical problem and those in the fifth group underwent a tone detection procedure. Individuals in the sixth group served as a control and were not subjected to an overt interfering activity. Results confirmed that any form of interfering task significantly decreased recall in relation to the control group, but there were no differences between the five interference conditions. This suggests that any task requiring mental effort may cause RI and damage recall, and subsequent research has shown that spot-the-difference puzzles can interfere with memory for verbal materials, such as stories (e.g., Dewar et al., 2012) and nonrecallable nonwords (Dewar et al., 2014). Even internal events like autobiographical thinking may act as a source of RI before new representations have been consolidated (Craig, Della Sala, \& Dewar, 2014).

To date, there has been little effort to investigate nonspecific RI in children, but as children generally perform worse than adults on different memory tasks (see Gathercole, 1998, 1999) and are more likely to commit different types of memory errors (Ackil \& Zaragoza, 1995, 1998; Roberts, 2002), they might be especially vulnerable to this form of interference. Alternatively, whilst children are known to be susceptible to similarity-based RI (e.g., Darby \& Sloutsky, 2015; Lee \& Bussey, 2001), they might be capable of resisting nonspecific RI in a manner similar to that of adults. Evidence for this possibility came from Darby and Sloutsky (2013, Experiment 2), who found that dissimilar interfering events have no effect on recall. Children and adults learnt object pairings and then attempted to predict the location at which an object would reemerge after entering an occluder. The first phase trained participants to perform the task and the final phase incorporated the memory test, with the middle component including a simple distractor task. During this task, two arrows were presented on each trial and participants predicted their direction when they reemerged from the occluder. This was designed to be a straightforward task that did not introduce new learning. Results revealed an improvement in performance during the final memory test, suggesting that the intervening activity did not impair memory for the object pairs in any way. Whilst 
the results of Darby and Sloutsky (2013) suggest children can resist nonspecific RI, there is need for caution. This form of interference may only be manifested if the distractor task requires some cognitive effort, yet Darby and Sloutsky's (2013) intervening activity was very easy and performance exceeded $90 \%$ for both children and adults.

In summary, the effects of nonspecific RI on children remain unclear. Few previous studies directly aimed to investigate this form of interference, and the relevant existing experiments have tended to use variants of the A-B A-C paradigm. Whilst this methodology is a valid tool for assessing RI, it does provide a substantial amount of training and nonspecific RI may cease to have any effects in well-learnt tasks. Furthermore, many real world tasks may not involve interference that is highly similar to the original learning, but instead may include varied forms of cognitive activity that occur shortly after encoding. Consequently, nonspecific RI may be a more important source of memory loss in everyday activities than similarity-based RI (Wixted, 2004, 2005).

The first reported experiment aimed to examine the impact of nonspecific RI in children (aged 6 or 7), with a sample of adults being recruited for comparison. The basic procedure was inspired by Dewar et al. (2007) and involved a word recall test. Participants were presented with a list of words followed by a recall test 5 min later. During the interval, participants either waited quietly (the control condition) or attempted a series of spot-the-difference puzzles (the interference condition). The spot-the-difference activity-which was dissimilar to the original word list-was predicted to produce nonspecific RI and damage subsequent word recall (see Dewar et al., 2007). Both children and adults were expected to recall fewer words in the interference condition than the control condition, yet children were expected to be particularly vulnerable to the damaging influence of nonspecific RI.

\section{EXPERIMENT 1}

\section{Method}

\section{PARTICIPANTS}

Fifty-nine children (30 female) aged between 6 and $7\left(M_{\text {age }}=6.37\right.$, $S D=0.49$ ) were recruited from two primary schools in England. None had any reported special education needs. Sixty adults (40 female) also took part. The adults were aged between 18 and $61\left(M_{\text {age }}=26.93, S D\right.$ $=13.15$ ) and included a mixture of undergraduate students and members of the public.

\section{MATERIALS}

Two 20-item word lists were created using the Bristol Norms database (Stadthagen-Gonzalez \& Davis, 2006). The chosen words were between four and seven letters in length, but had age of acquisition scores varying between 4 and 4.5 years. As such, the children were expected to be familiar with the words that were used. In addition, the two lists were approximately equal in terms of mean age of acquisition ( $M=4.25$ for List $1 ; M=4.25$ for List 2$)$, imageability $(M=539$ for List
1; $M=535.1$ for List 2$)$ and familiarity $(M=568.3$ for List $1 ; M=562$ for List 2).

Twelve spot-the-difference puzzles were used in the distractor task. These were adapted from online sources and each puzzle contained two similar cartoons, with 12 subtle differences between the two images. The puzzles were shown in black and white and included the following: (a) a Halloween scene with a witch, pumpkin and animals, (b) an office space, (c) a Valentine's scene with a man offering a woman some flowers, (d) a birthday scene with an adult and child eating a cake, (e) a girl leaving a school bus, (f) two giraffes eating leaves, (g) some flowers, (h) a Christmas scene with a girl receiving a gift from Santa Claus, (i) a boy standing outside in the snow, (j) two children constructing a sand castle, (k) an array of 16 emoticons, and (l) a family singing a Christmas carol. This activity was chosen due to its visual nature and was therefore expected to be distinct to the initial learning activity (see Dewar et al., 2007). Whilst this does not prevent participants from verbally encoding elements of the visual scenes, the manner in which the pictures were presented-two adjacent scenes-was designed to encourage rapid visual comparisons between the images. In addition, participants were looking for specific changes to certain visual details (such as alterations in shape or colour), or omissions. These changes were always presented in the same location in the two images, so participants could easily compare whether one detail matched that in another. Most importantly, the puzzles did not require new verbal learning, which might be expected to produce similarity-based interference. The present distractor task is therefore very different to that used in other interference paradigms such as the A-B A-C method. Yet the puzzles did require sustained attention, which was expected to produce nonspecific RI (Dewar et al., 2007). A computer and projector were used to display the words.

\section{DESIGN AND PROCEDURE}

The two variables of interest were the groups (children vs. adults) and condition (control vs. interference). As the latter variable was fully within groups, counterbalancing was used to alleviate order effects ${ }^{1}$. The sample was split in half and some of the participants completed the control condition followed by the interference condition, and the remaining individuals undertook conditions in the reverse order. The two word lists were equally distributed over the two conditions, such that half of the participants studied List A in the control condition and List $B$ in the experimental condition, and the other half experienced the reverse order.

Two schools were contacted with an invitation to take part in the study. The procedure was discussed with the head teachers and consent forms were sent to the parents of the children falling within the targeted age range. Parental consent was obtained for every child who took part and the study was conducted within a classroom at each school. Children also verbally gave their consent to take part. The procedure was explained to the children in terms of the three different stages involved (studying a word list, waiting and then recalling the words) and they were then shown the lists via a projector. Participants knew that they would be required to recall the word lists from the outset of 
the experiment. All words were shown simultaneously and participants had 2 min to study them. After this there was a 5-min retention interval followed by a free recall test lasting $2 \mathrm{~min}$. The second phase of the study followed exactly the same procedure to that already described, except a new list of words was used.

The critical manipulation concerned the events occurring within the retention interval-in the control condition, this interval was unfilled and participants were asked to remain silent, whereas in the experimental condition, participants attempted to complete as many of the puzzles as possible. The same procedure was employed for the adult participants, except they provided their own written consent.

\section{Results and Discussion}

Recall scores were computed for each participant, but children often made spelling errors. To avoid underestimating children's recall scores by treating misspelt words as an erroneous answer, incorrectly spelt words could be classed as a correct response if the two authors independently rated the answer as (a) being sufficiently accurate and (b) obviously relating to one of the items on the list.

The number of words recalled was calculated based on summing the number of correct responses. Adults were much better at recalling the words than children, but direct comparisons of the effect of interference was problematic due to a violation of the homogeneity of variance assumption in the interference condition (variance was reduced in children in comparison to adults, $F[1,117]=19.82, p<.001)$. To correct this problem, data were adjusted using the logarithmic $(\log 10)$ transformation (see Osborne, 2002) ${ }^{2}$. The data, shown in Figure 1, were then examined using a $2 \times 2$ (Group [children, adults] $\times$ Condition [interference, control]) mixed analysis of variance (ANOVA).

Adults $(M=0.87)$ performed better than children $(M=0.61), F(1$, $113)=44.64, M S E=0.09, p<.0001, \eta_{p}^{2}=.28$, and more words were recalled in the control $(M=0.79)$ than interference $(M=0.69)$ condition, $F(1,113)=12.26, M S E=0.04, p=.001, \eta_{\mathrm{p}}{ }^{2}=.10$. However, these effects were qualified by a significant interaction, $F(1,113)=5.12, M S E=0.04$, $p=.03, \eta_{\mathrm{p}}^{2}=.04$, which was interpreted using Holm-Šidàk adjusted

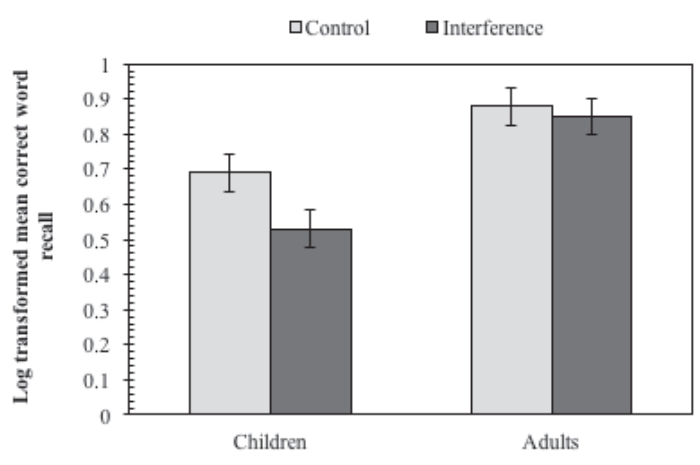

FIGURE 1.

Mean correct word recall (log transformed) in the control and interference conditions for children and adults. Error bars show 95\% Cls calculated according to Morey (2008). paired $t$ tests. In adults, there was no reliable difference between retention scores in the control and interference conditions, $t(58)=0.91, p$ $=.37, d=0.11$. Conversely, children, showed a significant decrease in retention when RI was present, in comparison to the control, $t(55)=$ $3.94, p<.001, d=0.53$.

To further explore the effects of RI, the Bayes factor $(B)$ was calculated according to Dienes (2014), and examined using the raw recall scores. This analysis allows evidence for null and alternative hypotheses to be directly compared, with a larger $B$ value indicating more support for the alterative than null hypothesis, and smaller $B$ values offering more support for the null. Interpretation of $B$ followed Dienes (2014) and Jeffreys (1961): Values of 0.33 or less show strong evidence for the null hypothesis, values of 3 or above offer strong evidence for the alternative hypothesis, and values in between represent insensitivity.

A uniform distribution was assumed, as forgetting could be quantified by monitoring changes in retention across the two conditions. Mean forgetting was computed by subtracting recall scores in the interference condition from those in the control condition. No forgetting would be represented by values of zero (i.e., no change from control to interference), whereas complete forgetting would be represented by values equivalent to the control (e.g., if retention in the control condition was six words, total loss of the representations following RI would lead to a score of zero in the experimental condition and a forgetting rate of six). Dienes' (2008) calculator was used to compute B (see http:// www.lifesci.sussex.ac.uk/home/Zoltan_Dienes/inference/bayes_factor.swf). In adults, there was substantial support for the null hypothesis (mean forgetting $=0.65$, lower bound $=0$, upper bound $=8.72$, sample $S E=0.52, B=0.29)$. In children, however, there was convincing evidence for the alternative hypothesis (mean forgetting $=2$, lower bound $=0$, upper bound $=5.53$, sample $S E=0.42, B=15980.39$ ).

The present results established a detrimental effect of nonspecific $\mathrm{RI}$ in children, with a large decrease in recall performance in the face of distraction, compared to a no-interference control. Whilst earlier studies had established the detrimental effect of similarity-based RI on children's memory (Darby \& Sloutsky, 2015; Howe, 1995, 2002, 2004; Lee \& Bussey, 2001), the current experiment showed that the interfering material does not need to resemble the learning material in order to exert an effect. Conversely, adults appeared to be unaffected by nonspecific RI. These results provide evidence that children-at least those aged between 6 and 7-are vulnerable to nonspecific RI, but this may only apply in cognitively demanding situations. Here, all participants were given $2 \mathrm{~min}$ to both encode and then later retrieve items, which the children may have found to be particularly difficult (although other studies of interference in children have presented stimuli at rapid rates, e.g., Levy-Gigi \& Vakil, 2010). Whilst the challenges of the memory task cannot fully account for the damaging effect of RI, given that the control condition had identical encoding and retrieval times, it is possible that children are able to better prevent interference when they are under less time pressure. This idea was tested in Experiment 2, and allowed an opportunity to assess whether children are capable of stopping interference in less challenging circumstances. 


\section{EXPERIMENT 2}

A new sample of children was recruited and their ability to encode and retrieve words was tested under interference and noninterference conditions. The arrangements were very similar to Experiment 1, but more time was given for encoding and retrieval. The aim was to assess whether children are better equipped to resist RI when the time constraints are relaxed. Given the results of Experiment 1, however, it was still expected that recall would be worse in the interference than control condition.

\section{Method}

\section{PARTICIPANTS}

Nineteen children (seven female) aged between 6 and $7\left(M_{\text {age }}\right.$ $=6.53, S D=0.51$ ) were recruited from another primary school in England. None of the children had participated in the first experiment and none were reported to have any special educational needs. Parental consent was obtained for all children who took part in the study and arrangements were approved by the class teacher.

\section{MATERIALS}

As in Experiment 1, two word lists were created. Each list contained 20 words selected from Key Stages 1 and 2, which is part of the national curriculum in England. Words varied between three and six letters in length, and the number of words of different lengths was constant for both lists $(M=4.15, S D=0.88)$. Words were rated as being high frequency for the age group (Department of Education, 2007). The intention was (a) to ensure all words were readily familiar to the participants, and (b) to reduce the number of spelling errors seen in Experiment 1.

\section{DESIGN AND PROCEDURE}

The arrangements for this experiment were very similar to those reported previously, with a few important alterations. The word lists were now shown for $5 \mathrm{~min}$, and an additional $5 \mathrm{~min}$ were given for recall. The ordering of the conditions was again counterbalanced, with nine children beginning with the control condition and 10 starting with the interference condition. This was designed to control for order effects $^{3}$. All other procedural arrangements matched that outlined for Experiment 1.

\section{Results and Discussion}

The number of spelling errors was relatively low, particularly in comparison to Experiment 1 (Experiment 2 spelling errors: $M=0.79, S D=$ 1.08; Experiment 1 spelling errors: $M=2.65, S D=1.80$ ). Nonetheless, spelling errors were treated in the same manner as outlined above. To investigate RI, data were assessed using the paired-samples $t$ test on the raw recall scores. Unlike Experiment 1, there was no reliable decrease in performance in the interference condition $(M=7.26, S D=2.33)$ compared with the control $(M=7.89, S D=2.71), t(18)=1.34, p=.20$, $d=0.31$. This suggests that when the demands of the memory task are eased, children can show some resistance to nonspecific RI.

To ensure the data were not simply insensitive-particularly given the reduced sample size in comparison with Experiment 1-another analysis using the Bayes factor was performed. This matched the arrangements highlighted above, with a uniform distribution being employed (mean forgetting $=0.63$, lower bound $=0$, upper bound $=7.89$, sample $S E=0.47)$. The corresponding $B$ value was 0.33 , which was on the boundary denoting strong support for the null hypothesis.

Under the arrangements of Experiment 2, children seemed better equipped to deal with nonspecific RI. This could have been due to the extended encoding and retrieval time, but it is also possible that children in this sample were simply better at memorising information and preventing interference than those recruited for Experiment 1. Overall mean recall for the children of Experiment $2(M=7.58, S D=2.30)$ was higher than that seen for the children in Experiment $1(M=6.53, S D$ =3.46), but there were high performers in the first study. To allow for a meaningful comparison across the two experiments, the children in Experiment 1 were placed into groups depending on their performance in the control condition. The highest scorers $\left(N=19^{4}\right)$-those in the top third of the sample-were then selected for further investigation (for these participants, mean recall in the control condition was similar to that recorded in Experiment 2). The data, shown in Figure 2, were analysed using a $2 \times 2$ (Experiment $[1,2] \times$ Condition [control, interference]) mixed ANOVA. There was no effect of the experiment, with the top scorers in the first study performing similarly to those in the second study, $F(1,36)=1.44, M S E=8.77, p=.24, \eta_{\mathrm{p}}{ }^{2}=.04$. There was a main effect of condition, $F(1,36)=56.86, M S E=2.00, p$ $<.001, \eta_{\mathrm{p}}{ }^{2}=.61$, with recall being worse following interference $(M=$ 5.95) than the silent control $(M=8.40)$, but this effect was qualified by a significant interaction, $F(1,36)=31.30, M S E=2.00, p<.001, \eta_{\mathrm{p}}{ }^{2}$ $=.47$. Follow-up independent $t$ tests, corrected for multiple comparisons using the Holm-Šidàk adjustment, found no difference in recall

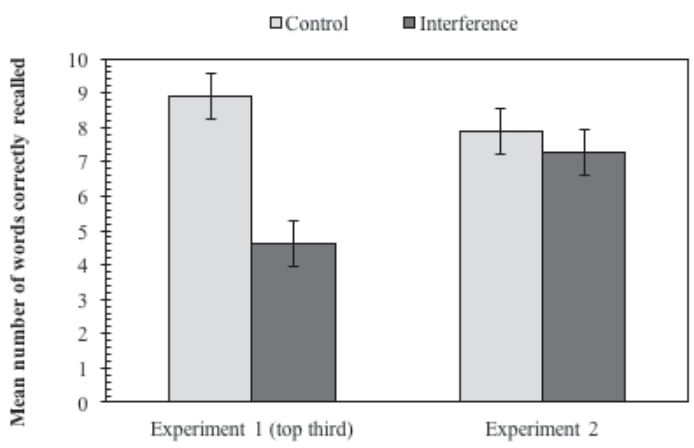

\section{FIGURE 2.}

Mean number of words correctly recalled in the control and interference conditions for the children in Experiments 1 and 2. The data from Experiment 1 shows the top third of the sample, due to similar performance in the control condition. Error bars show 95\% Cls for a mixed design calculated according to Jarmasz and Hollands (2009). 
between the two samples in the control condition, $t(36)=1.35, p=.19$, $d=0.45$. Conversely, children from Experiment 1 recalled significantly fewer words than those in Experiment 2 following interference, $t(36)$ $=-3.44, p=.001, d=-1.11$. Overall, the effects of RI were much more pronounced in Experiment 1 than Experiment 2, even when the children performed similarly in the control condition.

\section{GENERAL DISCUSSION}

The present experiment aimed to examine whether children are vulnerable to nonspecific RI. This project was motivated by the relatively sparse literature on nonspecific RI in children, even though this source of interference may be particularly prominent in everyday life (Wixted, 2004). The present procedure, incorporated into two experiments, involved two word recall tests. After being shown the words, there was a 5-min interval that was either silent (control condition) or contained a series of spot-the-difference puzzles (interference condition). A free recall test then occurred.

The results presented a more complex view of nonspecific RI, with the impact of interference changing according to the time available for encoding and retrieval. In Experiment 1, children showed significantly reduced word recall in the experimental condition in comparison with the no-interference control. Whilst children's susceptibility to RI is consistent with earlier studies (e.g., Howe, 1995, 2002, 2004; Lee \& Bussey, 2001), Experiment 1 showed that a distractor task that was highly dissimilar to the memory stimuli was particularly problematic for children, whereas adults were resilient to such interference. Whilst this latter finding contradicts some previous studies (e.g., Dewar et al., 2007), nonspecific RI in adults is generally thought to have a mild effect on retention (Dewar et al., 2010) and over short delays it appeared inconsequential.

The difference between adults and children could be due to variability in the effective use of retention strategies. Adult participants may have been better equipped to counter the detrimental effect of the spotthe-difference puzzles by using either covert rehearsal or attentional refreshing to remember the word list. There is some evidence suggesting that covert rehearsal and attentional refreshing strategies take time to develop (Gathercole, 1998; Tam, Jarrold, Baddeley, \& Sabatos-Devito, 2010) and fMRI evidence has also indicated developmental differences in verbal encoding (e.g., Menon, Boyett-Anderson, \& Reiss, 2005). Furthermore, children are less successful at using grouping techniques to remember information over the short-term (Cowan et al., 2006). This does not preclude the possibility that children can rehearse, but their ability to do so may be affected by capacity limitations (Cowan \& Vergauwe, 2014). It is acknowledged that such maintenance strategies are usually thought to operate over the short-term, but it seems likely that retention strategies would have been employed to some extent within the present experiment, although intermittently and perhaps less successfully in the children.

Importantly, however, the idea of qualitative differences in the retention strategies of children and adults has been questioned (see Miller, McCulloch, \& Jarrold, 2015) and Experiment 2 showed that nonspe- cific RI in children was absent. The core difference between the two experiments was the encoding and retrieval time (2 min in Experiment 1 vs. $5 \mathrm{~min}$ in Experiment 2). Whilst it is not possible to definitively determine whether encoding, retrieval, or a mixture of both processes was responsible for the resistance to RI, children rarely used the full timeframe to complete the retrieval test. The limited impact of RI in Experiment 2 may therefore have been due to improved encoding. If so, children may be capable of using strategies to resist nonspecific RI, but they need additional time to create effective and robust representations. This would suggest that children's mnemonic strategies are not necessarily undeveloped or unsophisticated, but they take longer to employ them. As they get older, children may become faster and more efficient at encoding information without prolonged study time, whilst still allowing successful defence against nonspecific RI.

The present study was primarily motivated to explore children's reactions to nonspecific RI, but these data raise some important questions concerning the underlying mechanisms of interference. The most common theoretical framework of nonspecific RI uses the consolidation process, in which newly formed memories are transformed into a stable and durable format. Nonspecific RI is hypothesised to interrupt this process, leading to forgetting (Dewar et al., 2012, 2014, 2007; Wixted, 2004). In Experiment 1, the limited encoding time may have impaired consolidation in children, leading to a stronger RI effect. Conversely, the extended encoding time in Experiment 2 may have improved the subsequent consolidation of the memories, helping children to resist nonspecific RI. Whilst this hypothesis needs further testing, consolidation does seem to play an important role in children's memory development (e.g., Backhaus, Hoeckesfeld, Born, Hohagen, \& Junghanns, 2008; Wilhelm, Prehn-Kristensen, \& Born, 2012), although consolidation theory does have a controversial standing within cognitive psychology (see Ecker \& Lewandowsky, 2012). RI could act independently from the consolidation process (Mercer, 2015) and the longer encoding time may itself have been responsible for the reduced forgetting in Experiment 2.

An alternative interpretation of the interference effect relies on disrupted maintenance. Specifically, the distractor activity may have prevented the employment of maintenance strategies, such as rehearsal, in children (at least in Experiment 1). As noted above, the notion of maintenance can potentially account for some of the findings recorded here and it is plausible that participants attempted to deliberately maintain the memory of the word list across the delay, at least to some extent. Much of the existing research that has examined maintenance strategies in children has used working memory tasks with very short delays, but there is some indirect evidence consistent with the idea that children can rehearse or refresh information over delays similar to those used here (e.g., Mahy \& Moses, 2011). Nonetheless, some of the most recent evidence suggests that nonverbal tasks may not interfere with maintenance strategies. Mahy, Mohun, Müller, and Moses (2016) explored prospective memory using a two-part card-based task. The children had to tell a fictional character about the position of a red dot on each card (the ongoing task) and remember to hide any card featuring an animal in a box (the prospective memory task). After 
being familiarised with the task, a 3-min interval commenced. Verbal interference was introduced by asking the children a series of questions during the interval, whereas in the control condition, children quietly drew pictures (this was designed to remove verbal interference). The card game then commenced, with the number of correct responses to animal-based cards being recorded. Of most relevance to the present study, performance in the control condition was not associated with measures of verbal ability, whereas in the verbal interference condition it was. This suggests that a visual-based distractor activity did not prevent verbal rehearsal in a group of children younger than those used in the present study, and the children recruited in Experiment 1 may have been able to both rehearse the words and perform the spot-thedifference puzzles over the delay.

There are, of course, important differences between Mahy et al. (2016) and the present experiments, particularly in terms of the memory task and stimuli. The rehearsal interpretation of nonspecific RI therefore cannot be discarded and researchers aiming to understand the mechanisms of nonspecific RI would be advised to test both consolidation and maintenance/rehearsal explanations. The challenge will be to find a distractor task that does not interfere with maintenance strategies yet is sufficiently demanding to act as a source of interference.

In conclusion, nonspecific RI can affect children. Importantly though, children are able to prevent this form of interference when encoding and retrieval arrangements are less demanding, suggesting they do have the ability to retain information across interference-filled delays. Future research could examine developmental changes in interference resistance using longitudinal designs, and greater consideration could be given to nonspecific RI as a source of memory loss in children.

\section{FOOTNOTES}

${ }^{1}$ To assess whether order effects were present in Experiment 1, twoway mixed analyses of variance (ANOVAs), with variables including condition (control vs. interference) and order (control-interference vs. interference-control), were carried out. These analyses were performed separately for children and adults. For both age groups, the order of conditions did not have an effect, $F(1,57)=0.10, p=.76, \eta_{\mathrm{p}}{ }^{2}=.002$, for children, $F(1,58)=1.48, p=.23, \eta_{\mathrm{p}}^{2}=.03$, for adults, nor did order reliably interact with the condition variable itself, $F(1,57)=2.42, p=$ $.13, \eta_{\mathrm{p}}{ }^{2}=.04$, for children, $F(1,58)=1.72, p=.20, \eta_{\mathrm{p}}{ }^{2}=.03$, for adults.

${ }^{2}$ It was not possible to logarithmically transform recall scores for participants who had failed to remember any words in a condition (i.e., those who scored zero). This affected three children and one adult, leaving 56 participants in the former group and 59 participants in the latter group.

${ }^{3}$ Another two-way mixed ANOVA was used to examine order effects in Experiment 2. As before, the ANOVA included condition and order as variables. There was no effect of condition, $F(1,17)=0.20, p=$ $.66, \eta_{\mathrm{p}}^{2}=.01$, and no interaction, $F(1,17)=0.35, p=.57, \eta_{\mathrm{p}}^{2}=.02$.

${ }^{4}$ This manipulation did not lead to major imbalances in the original counterbalancing. The high scoring group from Experiment 1 included nine children who participated in the control condition first and 10 who began with the experimental condition.

\section{REFERENCES}

Ackil, J. K., \& Zaragoza, M. S. (1995). Developmental differences in eyewitness suggestibility and memory for source. Journal of Experimental Child Psychology, 60, 57-83. doi: 10.1006/ jecp.1995.1031 WWW

Ackil, J. K., \& Zaragoza, M. S. (1998). Memorial consequences of forced confabulation: Age differences in susceptibility to false memories. Developmental Psychology, 34, 1358-1372. doi: 10.1037/0012-1649.34.6.1358 WWW

Backhaus, J., Hoeckesfeld, R., Born, J., Hohagen, F., \& Junghanns, K. (2008). Immediate as well as delayed post learning sleep but not wakefulness enhances declarative memory consolidation in children. Neurobiology of Learning and Memory, 89, 76-80. doi: 10.1016/j.nIm.2007.08.010 $\overline{\mathrm{WWW}}$

Cowan, N., Elliott, E. M., Saults, J. S., Nugent, L. D., Bomb, P., \& Hismjatullina, A. (2006). Rethinking speed theories of cognitive development: Increasing the rate of recall without affecting accuracy. Psychological Science, 17, 67-73. doi: 10.1111/j.14679280.2005.01666.x WWW

Cowan, N., \& Vergauwe, E. (2014). Applying how adults rehearse to understand how rehearsal may develop. Frontiers in Psychology, 5:1538. doi: 10.3389/fpsyg.2014.01538 |wWW

Craig, M., Della Sala, S., \& Dewar, M. (2014). Autobiographical thinking interferes with episodic memory consolidation. PLOS ONE, 9:e93915. doi: 10.1371/journal.pone.0093915 |wWW

Darby, K., \& Sloutsky, V. M. (2013). Proactive and retroactive interference effects in development. In M. Knauff, M. Pauen, N. Sebanz, \& I. Wachsmuth (Eds.), Proceedings of the 35th Annual Conference of the Cognitive Science Society (pp. 2130-2135). Austin, TX: Cognitive Science Society.

Darby, K. P., \& Sloutsky, V. M. (2015). The cost of learning: Interference effects in memory development. Journal of Experimental Psychology: General, 144, 410-431. doi: 10.1037/ xge0000051 $\underline{\underline{\omega W}}$

Davidson, M. C., Amso, D., Anderson, L. C., \& Diamond, A. (2006). Development of cognitive control and executive functions from 4 to 13 years: Evidence from manipulation of memory, inhibition, and task switching. Neuropsychologia, 44, 2037-2078. doi: 10.1016/j.neuropsychologia.2006.02.006 [WWW

Department of Education (2007). Letters and sounds: Principles and practice of high quality phonics. Retrieved from https:// www.gov.uk/government/publications/letters-and-soundsprinciples-and-practice-of-high-quality-phonics-phase-oneteaching-programme

Dewar, M., Alber, J., Butler, C., Cowan, N., \& Della Sala, S. (2012). Brief wakeful resting boosts new memories over the long term. Psychological Science, 23, 955-960. doi: $10.1177 / 0956797612441220 \underline{\underline{W W}}$ 
Dewar, M., Alber, J., Cowan, N., \& Della Sala, S. (2014). Boosting long-term memory via wakeful rest: Intentional rehearsal is not necessary, consolidation is sufficient. PLOS ONE, 9:e109542. doi: 10.1371/journal.pone.0109542 $\overline{\mathrm{WWW}}$

Dewar, M. T., Cowan, N., \& Della Sala, S. (2007). Forgetting due to retroactive interference: A fusion of Muller and Pilzecker's (1900) early insights into everyday forgetting and recent research on anterograde amnesia. Cortex, 43, 616-634. doi: 10.1016/S0010-9452(08)70492-1 WwW

Dewar, M., Cowan, N., \& Della Sala, S. (2010). Forgetting due to retroactive interference in anterograde amnesia. In S. Della Sala (Ed.), Forgetting (pp. 185-209). Hove, UK: Psychology Press.

Dienes, Z. (2008). Understanding Psychology as a Science: An Introduction to Scientific and Statistical Inference. New York: NY: Palgrave Macmillan.

Dienes, Z. (2014). Using Bayes to get the most out of non-significant results. Frontiers in Psychology, 5:781. doi: 10.3389/ fpsyg.2014.0078 WWW

Ecker, U. K. H., \& Lewandowsky, S. (2012). Computational constraints in cognitive theories of forgetting. Frontiers in Psychology, 3:400. doi: 10.3389/fpsyg.2012.00400 |WWW

Gathercole, S. E. (1998). The development of memory. Journal of Child Psychology and Psychiatry, 39, 3-27. doi: 10.1017/ S0021963097001753 WWW

Gathercole, S. E. (1999). Cognitive approaches to the development of short-term memory. Trends in Cognitive Sciences, 3, 410-419. doi: 10.1016/S1364-6613(99)01388-1 WWW

Hardt, O., Nader, K., \& Nadel, L. (2013). Decay happens: The role of active forgetting in memory. Trends in Cognitive Sciences, 17, 111-120. doi: 10.1016/j.tics.2013.01.001 |WW

Howe, M. L. (1995). Interference effects in young children's longterm retention. Developmental Psychology, 31, 579-596. doi: 10.1037/0012-1649.31.4.579

Howe, M. L. (2002). The role of intentional forgetting in reducing children's retroactive interference. Developmental Psychology, 38, 3-14. doi: 10.1037/0012-1649.38.1.3 WWW

Howe, M. L. (2004). The role of conceptual recoding in reducing children's retroactive interference. Developmental Psychology,

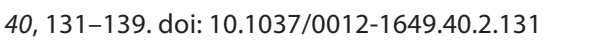

Jarmasz, J., \& Hollands, J. G. (2009). Confidence intervals in repeated-measures designs: The number of observations principle. Canadian Journal of Experimental Psychology, 63, 124-138. doi: 10.1037/a0014164 WWW

Jeffreys, H. (1961). The theory of probability (3rd ed.). Oxford, England: Oxford University Press.

Keppel, G. (1968). Retroactive and proactive inhibition. In T. R. Dixon \& D. L. Horton (Eds.), Verbal behavior and general behavior theory (pp. 172-213). Englewood Cliffs, NJ: Prentice-Hall.

Koppenaal, R. J., Krull, A., \& Katz, H. (1964). Age, interference, and forgetting. Journal of Experimental Child Psychology, 1, 360-375. doi: 10.1016/0022-0965(64)90029-3
Lee, K., \& Bussey, K. (2001). Children's susceptibility to retroactive interference: The effects of age and degree of learning. Journal of Experimental Child Psychology, 80, 372-391. doi: 10.1006/ jecp.2001.2638 WWW

Levy-Gigi, E., \& Vakil, E. (2010). Developmental differences in the impact of contextual factors on susceptibility to retroactive interference. Journal of Experimental Child Psychology, 105, 51-62. doi: 10.1016/j.jecp.2009.09.002 WWW

Loomis, D. J., \& Hall, V. C. (1968). The effects of age and overlearning in an A-B A-C design using high- and low-H value. Journal of Verbal Learning \& Verbal Behavior, 7, 182-188. doi: 10.1016/ S0022-5371(68)80186-0

Mahy, C. E. V., Mohun, H., Müller, U., \& Moses, L. J. (2016). The role of subvocal rehearsal in preschool children's prospective memory. Cognitive Development, 39, 189-196. doi: 10.1016/j. cogdev.2016.07.001

Mahy, C. E. V., \& Moses, L. J. (2011). Executive functioning and prospective memory in young children. Cognitive Development, 26, 269-281. doi: 10.1016/j.cogdev.2011.06.002

McGeoch, J. A. (1931). The influence of four different interpolated activities upon retention. Journal of Experimental Psychology, 14, 400-413. doi: 10.1037/h0074435

Menon, V., Boyett-Anderson, J. M., \& Reiss, A. L. (2005). Maturation of medial temporal lobe response and connectivity during memory encoding. Cognitive Brain Research, 25, 379-385. doi: 10.1016/j.cogbrainres.2005.07.007 WWW

Mercer,T. (2015).Wakeful rest alleviates interference-based forgetting. Memory, 23, 127-137.doi:10.1080/09658211.2013.872279 WWW

Miller, S., McCulloch, S., \& Jarrold, C. (2015). The development of memory maintenance strategies: Training cumulative rehearsal and interactive imagery in children aged between 5 and 9. Frontiers in Psychology, 6:524. doi: 10.3389/fpsyg.2015.00524 WWW

Morey, R. D. (2008). Confidence intervals from normalized data: A correction to Cousineau (2005). Tutorialsin QuantitativeMethods for Psychology, 4, 61-64. doi: 10.20982/tqmp.04.2.p061

Müller, G. E., \& Pilzecker, A. (1900). Experimentelle Beiträge zur Lehre vom Gedächtnis [Experimental contributions to the science of memory]. Zeitschrift für Psychologie, 1, 1-300.

Murre, J. M. J., \& Dros, J. (2015). Replication and analysis of Ebbinghaus' forgetting curve. PLOS ONE, 10:e0120644. doi:

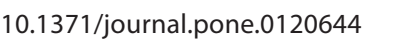

Nairne, J. S., \& Pandeirada, J. N. S. (2008). Forgetting. In H. L. Roediger, III. (Ed.), Cognitive psychology of memory. Learning and memory: A comprehensive reference (Vol. 2, pp. 179-194). Oxford, England: Elsevier.

Osborne, J. W. (2002). Notes on the use of data transformations. Practical Assessment, Research \& Evaluation, 8, 1-5. Retrieved from http://pareonline.net/getvn.asp? $v=8 \& n=6$

Roberts, K. P. (2002). Children's ability to distinguish between memories from multiple sources: Implications for the quality 
and accuracy of eyewitness statements. Developmental Review, 22, 403-435. doi: 10.1016/S0273-2297(02)00005-9

Robinson, E. S. (1920). Studies from the psychological laboratory of the University of Chicago: Some factors determining the degree of retroactive inhibition. Psychological Monographs, 28, 1-57. doi: 10.1037/h0093155

Stadthagen-Gonzalez, H., \& Davis, C. J. (2006). The Bristol norms for age of acquisition, imageability, and familiarity. Behavior Research Methods, 38, 598-605. doi: 10.3758/BF03193891 |www|

Tam, H., Jarrold, C., Baddeley, A. D., \& Sabatos-DeVito, M. (2010). The development of memory maintenance: Children's use of phonological rehearsal and attentional refreshment in work- ing memory tasks. Journal of Experimental Child Psychology, 107, 306-324. doi: 10.1016/j.jecp.2010.05.006 WWW

Wilhelm, I., Prehn-Kristensen, A., \& Born, J. (2012). Sleepdependent memory consolidation-What can be learnt from children? Neuroscience \& Biobehavioral Reviews, 36, 1718-1728. doi: 10.1016/j.neubiorev.2012.03.002 [WwW

Wixted, J. T. (2004). The psychology and neuropsychology of forgetting. Annual Review of Psychology, 55, 235-269. doi: 10.1146/annurev.psych.55.090902.141555

Wixted, J. T. (2005). A theory about why we forget what we once knew. Current Directions in Psychological Science, 14, 6-9. doi: 10.1111/j.0963-7214.2005.00324.x

RECEIVED 23.06.2017| ACCEPTED 02.11.2017 